\title{
Spectrum Sharing in Cognitive Radio Systems
}

\author{
Raouia Masmoudi \\ Gaspard Monge Computer Laboratory (LIGM) \\ University Paris-Est Marne-la-Vallée, France \\ Email: raouia.masmoudi@u-pem.fr
}

\begin{abstract}
In this paper, we focus on a wireless flat fading channel in a Cognitive radio network composed of licensed users (primary) and unlicensed users (secondary). We propose a framework for analyzing the access strategy based on the received interference at the secondary receiver and analyzing the achievable capacity of the secondary under the average power and average interference constraints. The proposed approach to the spectrum sharing, based on the received interference at the secondary receiver, significantly reduces the system complexity. We categorize the spectrum sharing into two cases based on the maximum interference threshold. We obtain a critical system parameter to eliminate the interference threshold constraint.

Index Terms-Cognitive radio systems, access strategy, achievable capacity, interference threshold, spectrum sharing.
\end{abstract}

\section{INTRODUCTION}

Fixed spectrum allocation to licensed users are inefficient in terms of spectrum utilization [1]. According to [2] and [3], the Cognitive Radio (CR) concept allows opportunistic users to exploit the underutilized spectrum. Primary users (PUs) are the licensed users who own the spectrum. However, secondary users (SUs) are unlicensed users tolerated in the primary spectrum provided they do not interfere too much with the primary users. In spectrum sharing, a SU is able to access to the PU's spectrum [3], [4]. Various schemes are proposed in the literature for spectrum sharing (see, e.g., [5]).

In this paper, we focus on the achievable capacity of the SU in spectrum sharing in an underlay access strategy. The achievable capacity is studied in [6] under various fading conditions. In [7], the impact of the interference threshold constraint on the achievable capacity of the SU is studied. In our scenario of interest, the SU can always access to the spectrum subject to the interference threshold constraint. Satisfying interference threshold constraint is technically challenging, since the SU requires the amount of interference power received at the primary receiver. Moreover, in the underlay strategy, the SU must satisfy the interference threshold even if the primary transmitter is inactive.

The main contribution of this paper is to propose a framework for analyzing the underlay access strategy based on the received interference at the secondary receiver, $I$, and analyzing the achievable capacity of the SU. The proposed approach to the spectrum sharing based on $I$ significantly reduces the system complexity comparing to the system in

This work is funded by Gaspard-Monge Computer Science Laboratory (LIGM) during my position as a researcher and teaching assistant at University of Paris-Est Marne-la-Valle (UPEM), France. which for spectrum sharing, the imposed interference at the primary receiver is required. In many previous works, the impact of $I$ on the secondary service performance is not considered (see, e.g., [6], [8], [9]). The main objective of this paper is to evaluate the maximum achievable capacity of the SU under underlay access strategy.

The rest of this paper is organized as follows: In Section II, the system model is presented; then, in Section III, the optimization problem in underlay spectrum sharing is analyzed. In Section IV, we solve this problem, calculate the power allocation and the achievable capacity of the SU. Consequently, in Section V, we present some interesting simulation results. The paper is concluded in Section VI.

\section{SySTEM MOdEL}

In this section, we describe the system model used in this paper. We consider a wireless flat fading WGN channel in Cognitive Radio (CR) systems. Two types of users try to access primary spectrum: Primary users (PUs) and Secondary users (SUs). In this context, the spectrum has been licensed to the primary users. The secondary users do not own the spectrum license; however, they may acquire access to the spectrum based on Opportunistic Spectrum Access (OSA) strategy. We assume that there is no direct signalling channel between PUs and SUs, and PUs is not aware of the presence of the SUs. ${ }^{1}$

In this paper, as shown in Fig. 1(a), we consider a spectrum sharing scenario with a primary transceiver and a secondary transceiver denoted by PT/PR and ST/SR, respectively. We represent in Fig. 1(a) the spectrum sharing system, in the CR context, considered in this paper. In each time instant $n, g_{s p}[n]$ and $g_{s s}[n]$ denote the instantaneous channel power gains from ST to PR and from ST to SR, respectively. Similarly, we define $g_{p s}[n]$ and $g_{p p}[n]$ as the instantaneous channel power gains from PT to SR and from PT to PR, respectively [10].

We assume that the quasi-static Rayleigh fading is present, and the channel model is abstracted in Fig. 1(b). At a specific time $n$, the received signals for both the primary receiver and the secondary receiver are:

$$
\begin{aligned}
& Y_{s}[n]=\sqrt{g_{s s}[n]} X_{s}[n]+\sqrt{g_{p s}[n]} X_{p}[n]+N_{s}[n], \\
& Y_{p}[n]=\sqrt{g_{s p}[n]} X_{s}[n]+\sqrt{g_{p p}[n]} X_{p}[n]+N_{p}[n],
\end{aligned}
$$

where $X_{s}$ and $X_{p}$ are the transmitted signals from ST and PT at time $n$, respectively. We denoted by $N_{s}$ and $N_{p}$ the additive

\footnotetext{
${ }^{1}$ We denote by the subscripts $s$ and $p$ to refer to SUs, and PUs, respectively.
} 


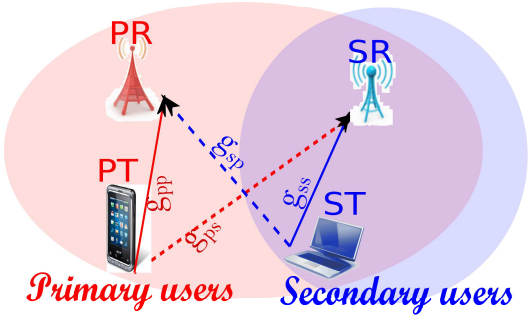

(a) Cognitive radio model

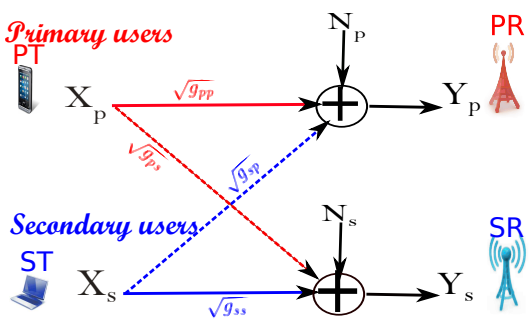

(b) The flat fading channel model

Fig. 1: System model for spectrum sharing in cognitive radio networks

white Gaussian noises at SR and PR with variances $\sigma_{s}^{2}=N_{0} B$ and $\sigma_{p}^{2}$, respectively. For reasons of clarity, hereafter, the time index $n$ is dropped. The power channel gains $g_{s s}, g_{s p}, g_{p p}$, and $g_{p s}$ are assumed to be stationary and ergodic independent random processes. For easy reference, the parameters utilized throughout the paper are also presented in the following Table:

\begin{tabular}{l|l}
\hline Parameters & Description \\
\hline$N_{0}$ & $\begin{array}{l}\text { power spectral density of the white noise } \\
\text { total bandwidth of the primary user } \\
\text { maximum transmit power of ST }\end{array}$ \\
$\bar{P}_{s}$ & $\begin{array}{l}\text { maximum interference threshold } \\
\text { critical interference threshold of } \bar{Q}\end{array}$ \\
$\gamma_{Q}^{*}$ & average imposed interference from PT to SR \\
$I$ & power gain of the channel between ST and SR \\
$g_{s s}$ & power gain of the channel between ST and PR \\
$g_{s p}$ & power gain of the channel between PT and SR \\
$g_{p s}$ & power gain of the channel between PT and PR \\
$g_{p p}$
\end{tabular}

In this paper, we focus on the underlay strategy. In the following, we investigate the corresponding secondary users achievable capacity for this underlay access strategy.

\section{Optimization Problem In Underlay ACCESS STRATEGY}

As it was mentioned before, in the underlay strategy, the primary user requires the secondary user to always satisfy the interference constraint at PR. Therefore, even in some cases when the primary user is not transmitting, the secondary user has to adjust its transmission power based on the interference threshold constraint. Therefore, in the underlay strategy, the secondary user must always keep the imposed interference at the primary receiver below this interference threshold; thus, requires to know the channel gains to adjust its transmission power.

\section{A. Maximum achievable capacity}

First, in our scenario, we define the signal to-interferenceand-noise ratio(SINR) for the the secondary receiver as follows:

$$
\gamma_{s}(\mathbf{g})=\frac{g_{s s} P_{s}}{N_{0} B+g_{p s} P_{p}}
$$

In the flat fading channel, the Shannon capacity is given by:

$$
C_{s h}=\mathbb{E}_{\mathbf{g}}\left[B \log \left(1+\gamma_{s}(\mathbf{g})\right)\right]
$$

where, $\mathbf{g} \triangleq\left(\left\{g_{s s}, g_{s p}\right\},\left\{g_{p s}, g_{p p}\right\}\right)=\left(\left\{\mathbf{g}_{s}\right\},\left\{\mathbf{g}_{p}\right\}\right)$ represents the instantaneous channel power gain and $\mathbb{E}_{\mathbf{x}}$ represents the expectation with respect to the random variable $\mathbf{x}$. Consequently using the previous equations (3) and (4), the maximum achievable capacity of the secondary user in the underlay strategy, $C_{U}$, is given by:

$$
C_{U}=\max _{P_{s} \geq 0} \mathbb{E}_{\mathbf{g}}\left[B \log \left(1+\frac{g_{s s} P_{s}}{N_{0} B+g_{s p} P_{p}}\right)\right]
$$

In $C_{U}, P_{s}$ is the transmission power allocated to ST which is generally a function of $g$. Similarly, $P_{p}$ is the transmission power of PT. In the spectrum sharing, the primary user adjusts its transmission power only based on its own channel power gain $g_{p p}$; therefore, $P_{p}$ is only a function of $g_{p p}$.

\section{B. Interference and power constraints}

Note that different constraints on the secondary user transmission power and primary user received interference are usually considered in the related literature, in order to obtain the secondary user capacity. We may consider constraints on the average or peak transmission power of the secondary user. Similarly, we can also consider constraint of the peak or average received interference at the primary user receiver [11].

In this paper, we consider the following constraints:

a) The average power constraint at ST:

$$
\mathbb{E}_{\mathbf{g}}\left[P_{s}\right] \leq \bar{P}_{s},
$$

where $\bar{P}_{s}$ represents the maximum transmit power threshold of ST.

b) The average interference constraint at PR:

$$
\mathbb{E}_{\mathbf{g}}\left[g_{s p} P_{s}\right] \leq \bar{Q},
$$

where $\bar{Q}$ represents the maximum tolerable average interference at PR which is called the interference threshold. Therefore, the transmitted power at ST should be adjusted so that the interference received at PR is always kept below this interference threshold $\bar{Q}$.

\section{Optimization problem formulation}

In this paper, we consider the average secondary user transmission power and average primary user received interference, with the objective of finding the optimal power allocation with 
the highest achievable capacity. Thus, $C_{U}$, is the solution of the following optimization Problem $\mathcal{O}_{U}$ :

$$
\begin{aligned}
C_{U}= & \max _{P_{s} \geq 0} \mathbb{E}_{\mathbf{g}}\left[B \log \left(1+\frac{g_{s s} P_{s}}{N_{0} B+g_{s p} P_{p}}\right)\right] \\
\text { s.t. } \quad & \mathbb{E}_{\mathbf{g}}\left[P_{s}\right] \leq \bar{P}_{s}, \\
& \mathbb{E}_{\mathbf{g}}\left[g_{s p} P_{s}\right] \leq \bar{Q},
\end{aligned}
$$

In $\mathcal{O}_{U}$, the objective function, (5), is Shannon's channel capacity. Moreover, (6) represents the maximum transmission power constraint at ST, and (7) represents the interference threshold constraint of PR.

Finding a solution to $\mathcal{O}_{U}$ is rather difficult. In the following, we propose a straightforward approach to find an equivalent problem to $\mathcal{O}_{U}$. Our analysis suggests an upper bound on the secondary user ergodic capacity for the underlay access strategy. As it was mentioned in Section II, the channel power gains are independent. We also note that $h(x)=\log \left(1+\frac{a}{b+x}\right.$ is a convex function for $a \geq 0, b \geq 0$, and $x \geq 0$. Therefore, using the Jensen's inequality on the objective function in $\mathcal{O}_{U}$, it can be seen that:

$$
\begin{aligned}
& \mathbb{E}_{\mathbf{g}}\left[B \log \left(1+\frac{g_{s s} P_{s}}{N_{0} B+g_{p s} P_{p}}\right)\right] \geq \\
& \mathbb{E}_{\mathbf{g}}\left[B \log \left(1+\frac{g_{s s} P_{s}}{N_{0} B+\mathbb{E}_{\mathbf{g}_{\mathbf{p}}}\left[g_{p s} P_{p}\right]}\right)\right] .
\end{aligned}
$$

By defining the average imposed interference from PT to SR: $I \triangleq \mathbb{E}_{\mathbf{g}_{\mathbf{p}}}\left[g_{p s} P_{p}\right]$, we obtain the following SU's capacity depending on $I$ :

$$
C_{U}(I) \triangleq \max _{P_{s} \geq 0} \mathbb{E}_{\mathbf{g}_{\mathrm{s}}}\left[B \log \left(1+\frac{g_{s s} P_{s}}{N_{0} B+I}\right)\right]
$$

Thus, it's easy to see from the inequality in (9) and the capacities definitions in equations (5) and (10) that we have, $C_{U} \geq C_{U}(I)$. Therefore, our initial optimization problem $\mathcal{O}_{U}$ can be approximated as follows:

Problem $\widehat{\mathcal{O}}_{U}$ :

$$
\begin{aligned}
C_{U}(I)= & \max _{P_{s} \geq 0} \mathbb{E}_{\mathbf{g}_{\mathbf{s}}}\left[B \log \left(1+\frac{g_{s s} P_{s}}{N_{0} B+I}\right)\right] \\
\text { s.t. } \quad & \mathbb{E}_{\mathbf{g}_{\mathbf{s}}}\left[P_{s}\right] \leq \bar{P}_{s}, \\
& \mathbb{E}_{\mathbf{g}_{\mathbf{s}}}\left[g_{s p} P_{s}\right] \leq \bar{Q},
\end{aligned}
$$

where, in this case, $P_{s}$ is a function of $g_{s}$ and $I$; However, $I$ does not depend on $P_{s}$.

Theorem 1. Using Lagrange multipliers approach for $\widehat{\mathcal{O}}_{U}$, we obtain the optimal ${ }^{2}$ transmission power $P_{s}^{*}$ which can be written as a water-filling type of solution:

$$
P_{s}^{*}=\left(\frac{1}{\lambda_{1}^{*}+\lambda_{2}^{*} g_{s p}}-\frac{N_{0} B+I}{g_{s s}}\right)^{+} .
$$

The Lagrangian coefficients, $\lambda_{1}^{*}$ and $\lambda_{2}^{*}$, are also obtained by replacing $P_{s}^{*}$ into (10) and (11) considering equality. Therefore, these Lagrange multipliers must satisfy the following equalities:

$\int_{\mathcal{G}_{s}}\left(\frac{1}{\lambda_{1}^{*}+\lambda_{2}^{*} g_{s p}}-\frac{N_{0} B+I}{g_{s s}}\right) p\left(g_{s s}\right) p\left(g_{s p}\right) d g_{s s} d g_{s p}=\bar{P}_{s}$,

\footnotetext{
${ }^{2}$ We denote by $(x)^{+}=\max (0, x)$.
}

and

$\int_{\mathcal{G}_{s}}\left(\frac{g_{s p}}{\lambda_{1}^{*}+\lambda_{2}^{*} g_{s p}}-g_{s p} \frac{N_{0} B+I}{g_{s s}}\right) p\left(g_{s s}\right) p\left(g_{s p}\right) d g_{s s} d g_{s p}=\bar{Q}$.

Here, $p\left(g_{s s}\right), p\left(g_{s p}\right)$ stand for the probability density functions of the power gains $g_{s s}, g_{s p}$, respectively, and $\mathcal{G}_{s} \triangleq$ $\left\{\mathbf{g}_{s}: \frac{g_{s s}}{\lambda_{1}^{*}+\lambda_{2}^{*} g_{s p}} \geq N_{0} B+I\right\}$.

The proof of this theorem is detailed in Appendix A.

\section{Power Allocation To The Secondary User}

As it was shown in (14), $P_{s}^{*}$ is a function of $g_{s p}$ among others parameters. In other words, to evaluate $P_{s}^{*}$, ST requires the channel state information (CSI) between itself and PR [12]. In practice, obtaining the optimal power allocation $P_{s}^{*}$ is very complex. Thus, we categorize the underlay spectrum sharing into two following cases:

- First, the case where the interference threshold $\bar{Q}$ is very high, and/or $\bar{P}_{s}$ is small enough so that the corresponding created interference at the front end of the primary user receiver never crosses the interference threshold, $\bar{Q}$. In such cases, the interference constraint in (13) is always satisfied. Thus, the dominant constraint is the maximum transmit power constraint of the secondary service in (12). Next, we will analyse this case in subsection IV-A.

- The second case, is the one where $\bar{P}_{s}$ is very high (i.e.,there is no practical power constraint for the secondary service), and/or the interference threshold $\bar{Q}$ is small enough so that for any feasible power allocation holds in (13), the power constraint in (12) is always satisfied. In this case, the dominant constraint is the interference threshold constraint in (13). This case will be analysed in details in subsection IV-B.

\section{A. Power constrained spectrum sharing}

In the power-constrained spectrum sharing, the interference threshold $\bar{Q}$ is always satisfied. Therefore, $\widehat{\mathcal{O}}_{U}$ is converted into the following optimization problem:

$$
\begin{aligned}
C_{U}^{P}(I)= & \max _{P_{s} \geq 0} \mathbb{E}_{\mathbf{g}_{\mathbf{s}}}\left[B \log \left(1+\frac{g_{s s} P_{s}}{N_{0} B+I}\right)\right] \\
\text { s.t. } & \mathbb{E}_{\mathbf{g}_{\mathbf{s}}}\left[P_{s}\right] \leq \bar{P}_{s} .
\end{aligned}
$$

The above optimization is an instance of water-filling problem [14], [15]. Similarly to Theorem 1 , by replacing $\lambda_{1}^{*}=\lambda_{P}^{*}$ and $\lambda_{2}^{*}=0$, the optimal transmission power in (14) becomes:

$$
P_{s}^{*}=\left(\frac{1}{\lambda_{P}^{*}}-\frac{N_{0} B+I}{g_{s s}}\right)^{+} .
$$

For the Rayleigh fading channel, $\lambda_{P}^{*}$ is the Lagrangian coefficient ${ }^{3}$ which is obtained from:

$$
\frac{\mathbf{H}_{1}\left(\rho_{P}\right)}{\rho_{P}}=\gamma_{P}
$$

${ }^{3}$ We denote by $\mathbf{E}_{1}(x)$ the exponential integral of order 1 defined as $\mathbf{E}_{1}(x) \triangleq \int_{1}^{\infty} t^{-1} e^{-x t} d t$ and the integral $\mathbf{H}_{1}(x)$ is given by $\mathbf{H}_{1}(x) \triangleq$ $\int_{1}^{\infty} \frac{e^{-x t}}{t^{2}} d t, x \geq 0$. 
where the parameters $\rho_{P}$ and $\gamma_{P}$ are defined as follows:

$$
\rho_{P} \triangleq \lambda_{P}^{*}\left(N_{0} B+I\right) \text { and } \gamma_{P} \triangleq \bar{P}_{s} /\left(N_{0} B+I\right) .
$$

Therefore, using equations (15) and (17), the achievable capacity of the secondary user is given by:

$$
C_{U}^{P}=B \mathbf{E}_{1}\left(\rho_{P}\right) .
$$

\section{$B$. Interference constrained spectrum sharing}

In the interference-constrained spectrum sharing, the power threshold $\bar{P}_{s}$ is always satisfied. Therefore, $\widehat{\mathcal{O}}_{U}$ is converted into the following optimization problem:

$$
\begin{aligned}
C_{U}^{Q}(I)= & \max _{P_{s} \geq 0} \mathbb{E}_{\mathbf{g}_{\mathbf{s}}}\left[B \log \left(1+\frac{g_{s s} P_{s}}{N_{0} B+I}\right)\right] \\
\text { s.t. } & \mathbb{E}_{\mathbf{g}_{\mathbf{s}}}\left[g_{s p} P_{s}\right] \leq \bar{Q} .
\end{aligned}
$$

The above optimization is an instance of water-filling problem. Similarly to Theorem 1 , by replacing $\lambda_{2}^{*}=\lambda_{Q}^{*}$ and $\lambda_{1}^{*}=0$, the optimal transmission power in (14) becomes:

$$
P_{s}^{*}=\left(\frac{1}{g_{s p} \lambda_{Q}^{*}}-\frac{N_{0} B+I}{g_{s s}}\right)^{+} .
$$

For the Rayleigh fading channel, $\lambda_{P}^{*}$ is the Lagrangian coefficient which is obtained from:

$$
\rho_{P}-\log \left(1+\rho_{P}\right)=\gamma_{Q},
$$

where the parameters $\rho_{Q}$ and $\gamma_{Q}$ are defined as follows:

$$
\rho_{Q} \triangleq \lambda_{Q}^{*}\left(N_{0} B+I\right) \text { and } \gamma_{Q} \triangleq \bar{Q} /\left(N_{0} B+I\right) .
$$

Therefore, using equations (21) and (23), the achievable capacity of the secondary user is given by:

$$
C_{U}^{Q}=B \log \left(1+\rho_{Q}\right) .
$$

As it is seen, the optimization problem $\widehat{\mathcal{O}}_{U}$ in (11), for interference-constrained, and power-constrained spectrum sharing systems, is reduced into simpler optimization problems (15) and (21), respectively. Thus, identifying the type of the spectrum sharing is critical for system parameters adjustments. As it was mentioned, the achievable capacity $C_{U}^{Q}(I)$ (see (26)) is an increasing function of the interference threshold $\bar{Q}$ (through $\gamma_{Q}$ ); however, $C_{U}^{P}(I)$ (see (20)) does not change with $\gamma_{Q}$. We define then $\gamma_{Q}^{*}$ the critical threshold which is the intersection point between the two achievable capacities $C_{U}^{P}(I)$ and $C_{U}^{Q}(I)$, for a given $I$.

- Therefore, for the amount of $\bar{Q}$ where $\gamma_{Q} \leq \gamma_{Q}^{*}$ the spectrum sharing system acts as an interference-constrained spectrum sharing system with the achievable capacity of the secondary user $C_{U}^{Q}(I)$.

- However, for the case that $\gamma_{Q} \geq \gamma_{Q}^{*}$, the spectrum sharing system acts as a power-constrained spectrum sharing with the achievable capacity $C_{U}^{P}(I)$.

This critical parameter $\gamma_{Q}^{*}$ can be obtained from (15), (18), (21) and (24) as follows:

$$
\gamma_{Q}^{*}=\gamma_{P}+\left(\rho_{Q}-\frac{e^{-\rho_{Q}}}{\rho_{Q}}\right) .
$$

\section{NUMERICAL RESULTS}

In this section, we evaluate our proposed method via numerical simulations. All observations below have been verified via extensive simulations with generic parameters. We have selected only a few of the most illustrative and interesting scenarios to be presented next. In the following, we consider the flat fading CR system with a single active PU and one SU. The bandwidth is $B=1000 \mathrm{~Hz}$, the noise and interference power is normalized as $N_{0}=0.1 \mathrm{~W}$. The SU's power gains $g_{s s}$ and $g_{s p}$ are generated by random distribution $g_{s} \backsim \mathcal{N}\left(0, \sigma_{s}^{2}\right)$ and the maximum transmit power threshold is $\bar{P}_{s}=N_{0} B$.

\section{A. Maximum achievable capacity versus $I / N_{0} B$}

In Fig. 2, we plot the maximum achievable capacity $C_{U}^{P}(I)$ and $C_{U}^{Q}(I)$ versus the normalized average imposed interference $I / N_{0} B$. We remark that in both cases the maximum achievable capacity is decreasing when the normalized average imposed interference $I / N_{0} B$ is increasing. Notice that the maximum achievable capacity in the case of interference constrained spectrum sharing $C_{U}^{Q}(I)$ has higher values comparing to the maximum achievable capacity in the case of power constrained spectrum sharing $C_{U}^{P}(I)$.

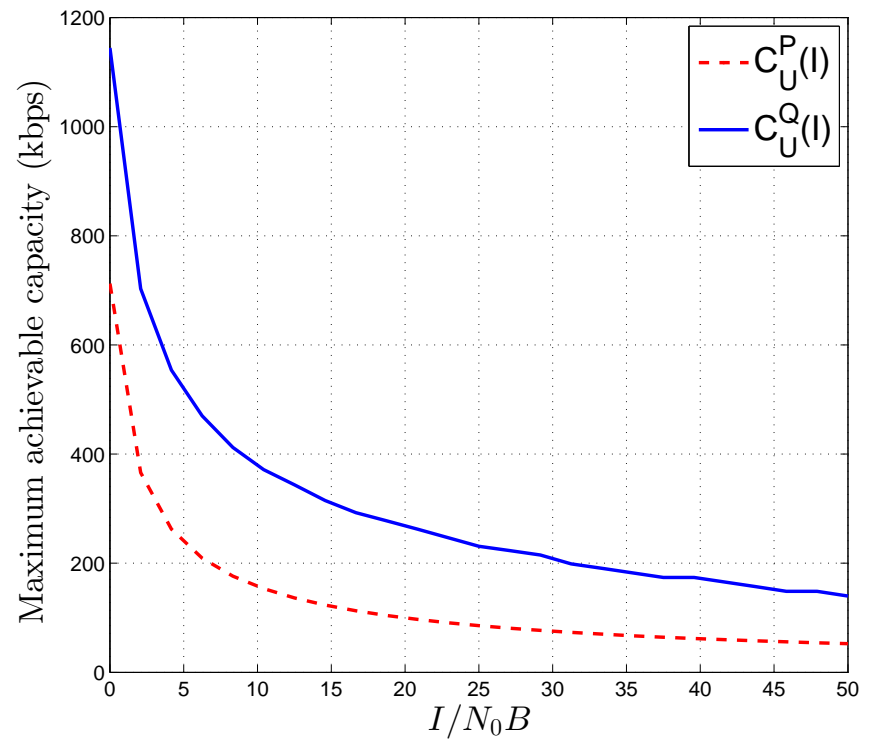

Fig. 2: The maximum achievable capacity of the SU, where $\bar{P}_{s}=\bar{Q}=N_{0} B$.

\section{B. Maximum achievable capacity versus $\gamma_{Q}^{*}$}

In Fig. 3, we consider the scenario in which the average imposed interference from PT to SR is $I=2 N_{0} B$. We plot the maximum achievable capacity of the SU versus the threshold $\gamma_{Q}$. We notice that there is a critical threshold $\gamma_{Q}^{*}=0.6$ which is the intersection point between the two achievable capacities $C_{U}^{P}(I)$ and $C_{U}^{Q}(I)$, for $I=2 N_{0} B$. Therefore, below this critical threshold, i.e., $\gamma_{Q} \leq \gamma_{Q}^{*}$, the spectrum sharing system acts as an interference-constrained spectrum sharing system with the achievable capacity $C_{U}^{Q}(I)$. However, above 


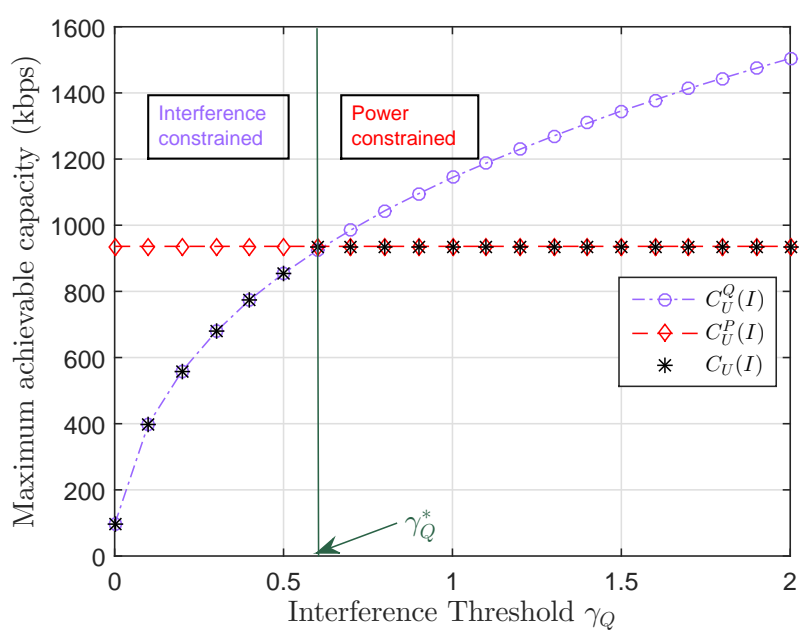

Fig. 3: The maximum achievable capacity of the SU for several values of $\gamma_{Q}$, where $I=2 N_{0} B$.

this critical threshold, i.e., $\gamma_{Q} \geq \gamma_{Q}^{*}$, the spectrum sharing system acts as a power-constrained spectrum sharing with the achievable capacity $C_{U}^{P}(I)$.

\section{Water-filling solution}

In Figure 4, we consider the previous scenario, in the case of 4 fading channels where the average imposed interference from PT to SR is $I=10 N_{0} B$ and the mean of the power gains $\sigma_{s}^{2}=2$. Thus, we represent the optimal power allocation as a water-filling type of solution in two cases which verifies respectively equation (17) and (23). We define by the Signal to Interference plus Noise Ratio (SINR) which is given by: $S I N R=\frac{g_{s s}}{N_{0} B+I}$. In the first case, i.e. $\gamma_{Q} \geq \gamma_{Q}^{*}$, we remark that the channel 2 and 3 are not allocated to SU. However, when $\gamma_{Q} \leq \gamma_{Q}^{*}$, the water level is not the same for all channels and then the channel 2 can be allocated to SU. This can be explained by the expression of the optimal power in (23) where the water level becomes function of $g_{s p}$.

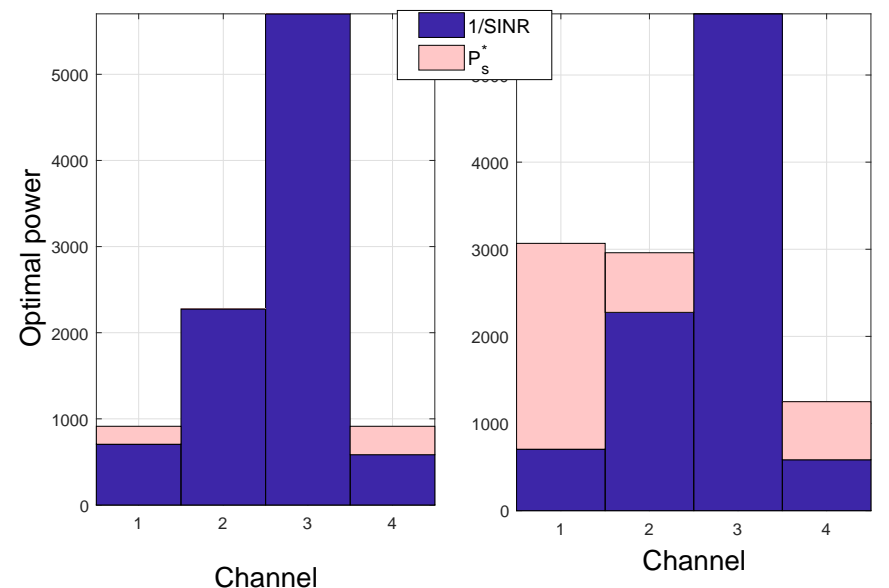

Fig. 4: Optimal power allocation is a water-filling solution (a) when $\gamma_{Q} \geq \gamma_{Q}^{*}$ and (b) when $\gamma_{Q} \leq \gamma_{Q}^{*}$.

\section{CONCLUSION}

In this paper, we analyzed the secondary achievable capacity for underlay access strategy under the power and interference constraints. We categorized this underlay spectrum sharing into two cases based on the maximum interference threshold. We obtained a critical system parameter to eliminate the interference threshold constraint which significantly reduce the system complexity by making the power allocation of the secondary user independent from the channel-side information between the secondary transmitter and the primary receiver. We have made this optimization problem under-study simpler enough so that we did not use an iterative algorithm to compute the optimal power allocation solution. Future works can include the analyzis of others access strategies for spectrum sharing systems (such as the overlay access strategy). Analyzing underlay access strategy with multiple primary and secondary users can be considered in the future as well [16].

\section{APPENDIX A}

\section{PROOF OF THEOREM 1}

As we define before the Problem $\widehat{\mathcal{O}}_{U}$ :

$$
\begin{aligned}
C_{U}(I)= & \max _{P_{s} \geq 0} \mathbb{E}_{\mathbf{g}_{\mathbf{s}}}\left[B \log \left(1+\frac{g_{s s} P_{s}}{N_{0} B+I}\right)\right] \\
\text { s.t. } \quad & \mathbb{E}_{\mathbf{g}_{\mathbf{s}}}\left[P_{s}\right] \leq \bar{P}_{s}, \\
& \mathbb{E}_{\mathbf{g}_{\mathbf{s}}}\left[g_{s p} P_{s}\right] \leq \bar{Q},
\end{aligned}
$$

Using the convex optimization tools [13], the Lagrangian can be written as:

$$
\begin{aligned}
\mathcal{L}\left(P_{s}, \lambda_{1}, \lambda_{2}\right) & =-\mathbb{E}_{\mathbf{g}_{\mathbf{s}}}\left[B \log \left(1+\frac{g_{s s} P_{s}}{N_{0} B+I}\right)\right] \\
& +\lambda_{1}\left(\mathbb{E}_{\mathbf{g}_{\mathbf{s}}}\left[P_{s}\right]-\bar{P}_{s}\right)+\lambda_{2}\left(\mathbb{E}_{\mathbf{g}_{\mathbf{s}}}\left[g_{s p} P_{s}\right]-\bar{Q}\right)
\end{aligned}
$$

where $\lambda_{1}$ and $\lambda_{2}$ are the Lagrangian coefficients, which satisfy respectively the power and interference constraints. Moreover, at the optimum, $\lambda_{1}^{*}$ and $\lambda_{2}^{*}$ are obtained by replacing the optimal power allocation into (29) and (30) considering equality. At the optimum, we have a zero derivative of Lagrangian on the optimal power allocation point:

$$
\left.\frac{\partial \mathcal{L}}{\partial P_{s}}\right|_{P_{s}=P_{s}^{*}}=0
$$

It is easy to see that: $\left.\frac{\partial}{\partial P_{s}}\left\{\mathbb{E}_{\mathbf{g}_{\mathbf{s}}}\left[P_{s}\right]\right\}\right|_{P_{s}=P_{s}^{*}}=1$, which means that:

$$
\frac{\partial}{\partial P_{s}}\left\{\mathbb{E}_{\mathbf{g}_{\mathbf{s}}}\left[B \log \left(1+\frac{g_{s s} P_{s}^{*}}{N_{0} B+I}\right)\right]\right\}=\lambda_{1}^{*}+\lambda_{2}^{*} \mathbb{E}_{\mathbf{g}_{\mathbf{s}}}\left[g_{s p}\right]
$$

As we know that the expectation $\mathbb{E}_{\mathbf{g}_{\mathbf{s}}}$ depends only on $\mathbf{g}_{\mathbf{s}}=$ $\left\{g_{s s}, g_{s p}\right\}$ and does not depend on $P_{s}$, we can then permute the partial derivative and $\mathbb{E}_{\mathbf{g}_{\mathrm{s}}}$, we obtain then:

$\mathbb{E}_{\mathbf{g}_{\mathrm{s}}}\left[B \frac{\partial}{\partial P_{s}}\left\{\log \left(1+\frac{g_{s s} P_{s}^{*}}{N_{0} B+I}\right)\right\}\right]=\mathbb{E}_{\mathbf{g}_{\mathbf{s}}}\left[\lambda_{1}^{*}+\lambda_{2}^{*} g_{s p}\right]$.

This is equivalent to:

$$
\mathbb{E}_{\mathbf{g}_{\mathrm{s}}}\left[B \frac{\frac{g_{s s}}{N_{0} B+I}}{1+\frac{g_{s s} P_{s}^{*}}{N_{0} B+I}}-\lambda_{1}^{*}-\lambda_{2}^{*} g_{s p}\right]=0
$$


From the previous equation, we obtain the optimal transmission power $P_{s}^{*}$ which can be written as:

$$
P_{s}^{*}=\left(\frac{1}{\lambda_{1}^{*}+\lambda_{2}^{*} g_{s p}}-\frac{N_{0} B+I}{g_{s s}}\right)^{+}
$$

In order to compute the Lagrangian coefficients, $\lambda_{1}^{*}$ and $\lambda_{2}^{*}$, we replace $P_{s}^{*}$ computed in (31) into (29) and (30) considering equality. Therefore, these Lagrange multipliers must satisfy the following equalities:

$$
\int_{g_{s s}} \int_{g_{s p}} P_{s}^{*} p\left(g_{s s}\right) p\left(g_{s p}\right) d g_{s s} d g_{s p}=\bar{P}_{s},
$$

and

$$
\int_{g_{s s}} \int_{g_{s p}} g_{s p} P_{s}^{*} p\left(g_{s s}\right) p\left(g_{s p}\right) d g_{s s} d g_{s p}=\bar{Q},
$$

where $p\left(g_{s s}\right), p\left(g_{s p}\right)$ represent the probability density functions of the power gains $g_{s s}, g_{s p}$, respectively. We define then the following set $\mathcal{G}_{s}$ :

$$
\mathcal{G}_{s} \triangleq\left\{\mathbf{g}_{s}: \frac{g_{s s}}{\lambda_{1}^{*}+\lambda_{2}^{*} g_{s p}} \geq N_{0} B+I\right\},
$$

which make $\left(\frac{1}{\lambda_{1}^{*}+\lambda_{2}^{*} g_{s p}}-\frac{N_{0} B+I}{g_{s s}}\right) \geq 0$, thus, we obtain:

$\int_{\mathcal{G}_{s}}\left(\frac{1}{\lambda_{1}^{*}+\lambda_{2}^{*} g_{s p}}-\frac{N_{0} B+I}{g_{s s}}\right) p\left(g_{s s}\right) p\left(g_{s p}\right) d g_{s s} d g_{s p}=\bar{P}_{s}$,

and

$\int_{\mathcal{G}_{s}}\left(\frac{g_{s p}}{\lambda_{1}^{*}+\lambda_{2}^{*} g_{s p}}-g_{s p} \frac{N_{0} B+I}{g_{s s}}\right) p\left(g_{s s}\right) p\left(g_{s p}\right) d g_{s s} d g_{s p}=\bar{Q}$.

\section{REFERENCES}

[1] Force FCC Spectrum Policy Task, "Report of the spectrum rights and responsibilities working group", technical report, Fed. Comm. Commission, Nov. 2002

[2] J. Mitola, "Cognitive Radio Architecture: The Engineering Foundations of Radio XML", Wiley Press, New York, 2006.

[3] S. Haykin, Cognitive Radio: Brain-Empowered Wireless Communications,IEEE J. Selected Areas in Comm., vol. 23, no. 2, pp. 201-220, Feb. 2005.

[4] J.M. Peha, "Approaches to Spectrum Sharing," IEEE Comm. Magazine, vol. 43, no. 2, pp. 10-12, Feb. 2005.

[5] Q. Zhao and B. Sadler, "A Survey of Dynamic Spectrum Access: Signal Processing, Networking, and Regulatory Policy," IEEE Signal Processing Magazine, vol. 24, no. 3, pp. 79-89, May 2007.

[6] A. Ghasemi and E.S. Sousa, "Fundamental Limits of SpectrumSharing in Fading Environments", IEEE Trans. Wireless Comm., vol. 6, no. 2, pp. 649-658, Feb. 2007.

[7] G. Bansal, M.J. Hossain, and V.K. Bhargava, "Optimal and Suboptimal Power Allocation Schemes for OFDM-Based Cognitive Radio Systems,"IEEE Trans. Wireless Comm.,vol. 7, no. 11, pp. 4710-4718, Nov. 2008

[8] L. Musavian and S. Aissa, "Capacity and Power Allocation for Spectrum-Sharing Communications in Fading Channels," IEEE Trans. Wireless Comm.,vol. 8, no. 1, pp. 148-156, Jan. 2009

[9] X. Kang, Y.C. Liang, H.K. Garg, and L. Zhang, "Sensing-Based Spectrum Sharing in Cognitive Radio Networks", IEEE Trans. Vehicular Technology, vol. 58, no. 8, pp. 4649-4654, Oct. 2009.

[10] R. Masmoudi, "Impact of Imperfect CSI on Resource Allocation in Cognitive Radio Channels", IEEE International Conference on Wireless and Mobile Computing, Networking and Communications (WiMob), Rome, Italy, 2017.
[11] M.S. Alouini and A.J. Goldsmith, "Capacity of Rayleigh Fading Channels under Different Adaptive Transmission and DiversityCombining Techniques", IEEE Trans. Vehicular Technology, vol. 48, no. 4, pp. 1165-1181, July 1999.

[12] R. Masmoudi, "A Realistic Error Model of Imperfect CSI in Cognitive Radio Channels", International Congress on Ultra Modern Telecommunications and Control Systems (ICUMT), Munich, Germany, 2017.

[13] S.Boyd and L. Vandenberghe, Convex Optimization, Cambridge Univ. Press, Cambridge, UK, 2004.

[14] R. Masmoudi, E. V. Belmega, I. Fijalkow, and N. Sellami, ”A closedform solution to the power minimization problem over two orthogonal frequency bands under QoS and cognitive radio interference constraints", IEEE DySPAN, pp. 212-222, USA, 2012.

[15] R. Masmoudi, E.V. Belmega , I. Fijalkow and N. Sellami, "A unifying view on energy-efficiency metrics in cognitive radio channels", European Signal Processing Conference, pp. 171 - 175, 2014.

[16] R. Masmoudi, E.V. Belmega , I. Fijalkow and N. Sellami, "Joint Scheduling and Power Allocation in Cognitive Radio Systems", IEEE International Workshop on Advances in Software Defined and Context Aware Cognitive Networks of IEEE International Conference on Communications (ICC), Londres, UK, 2015. 